\title{
Exact Formula of Probability and CP Violation for Neutrino Oscillations in Matter
}

\author{
K. Kimura ${ }^{1 *}$, A. Takamura ${ }^{1,2 \dagger}$, and H. Yokomakura ${ }^{1 \ddagger}$ \\ ${ }^{1}$ Department of Physics, Nagoya University, Nagoya, 464-8602, Japan \\ ${ }^{2}$ Department of Mathematics, Toyota National Collage of Technology \\ Eisei-cho 2-1, Toyota-shi, 471-8525, Japan
}

\begin{abstract}
Within the framework of the standard three neutrino scenario, we derive an exact and simple formula of the oscillation probability $P\left(\nu_{e} \rightarrow \nu_{\mu}\right)$ in constant matter by using a new method. From this formula, it is found that the matter effects can be separated from the pure $\mathrm{CP}$ violation effects. Furthermore, the oscillation probability can be written in the form, $P\left(\nu_{e} \rightarrow \nu_{\mu}\right)=A \cos \delta+B \sin \delta+C$, in the standard parametrization without any approximation. We also demonstrate that the approximate formula in high-energy can be easily reproduced from this as an example.
\end{abstract}

\section{Introduction}

Just like the quark system, it has been shown from the atmospheric neutrino experiments [1] and the solar neutrino experiments [2] that neutrinos have finite mass and finite mixing. In this situation, it is extremely interesting to investigate the $\mathrm{CP}$ phase in the lepton sector. Fortunately, recent report from SNO experiment [3] favors the LMA MSW solutions to the solar neutrino problem. This means that the measurements of $\mathrm{CP}$ phase may be possible because of the large 1-2 mixing angle and the large 1-2 mass difference.

In order to measure the $\mathrm{CP}$ phase, the long-baseline experiments such as the JHF experiment [4] and the neutrino factory experiments [5] are planned. In the past, the asymmetries $\Delta P_{C P}=$ $P\left(\nu_{\alpha} \rightarrow \nu_{\beta}\right)-P\left(\bar{\nu}_{\alpha} \rightarrow \bar{\nu}_{\beta}\right)$ and $\Delta P_{T}=P\left(\nu_{\alpha} \rightarrow \nu_{\beta}\right)-P\left(\nu_{\beta} \rightarrow \nu_{\alpha}\right)$ have been considered as the main approach to measure the CP phase $\delta[6,77,8,9]$. These are methods to measure the direct CP violation term which depends on $\sin \delta$. However, the measurement of $\Delta P_{C P}$ is not directly related to the discovery of $\mathrm{CP}$ phase, because of fake $\mathrm{CP}$ violating effects from the earth matter. $\Delta P_{T}$ is a pure $\mathrm{T}$ violating observable, but it has its own experimental difficulties. So, alternative approach has been recently considered in [10, 11, 12, 13, 14. This is an attempt to obtain the information on the $\mathrm{CP}$ phase totally from the probabilities itself, not only the direct $\mathrm{CP}$ violation term but also

\footnotetext{
${ }^{*}$ E-mail address:kimukei@eken.phys.nagoya-u.ac.jp

†E-mail address:takamura@eken.phys.nagoya-u.ac.jp

${ }^{\ddagger}$ E-mail address:yoko@eken.phys.nagoya-u.ac.jp
} 
the indirect $\mathrm{CP}$ violation term which depends on $\cos \delta$. In these papers the oscillation probability is written approximately in the form, $P\left(\nu_{e} \rightarrow \nu_{\mu}\right) \simeq A \cos \delta+B \sin \delta+C$. The extra information which is proportional to $\cos \delta$ will lead us to the value of $\delta$ in spite of the matter effect. In order to obtain more precise information, it is highly desirable to have an exact expression for $P\left(\nu_{e} \rightarrow \nu_{\mu}\right)$. Some attempts to derive the exact formula have been made in the context of three neutrino scenarios 15, 16, 17, 18]. These formulae are useful for numerical calculation. However, the precise CP dependence of $P\left(\nu_{e} \rightarrow \nu_{\mu}\right)$ has not been investigated sufficiently [16].

To describe our approach, let us review the work of Naumov [19] and Harrison-Scott [6]. The Hamiltonian $\tilde{H}$ in matter is related to $H$ in vacuum as

$$
\tilde{H}=H+\frac{1}{2 E} \operatorname{diag}(a, 0,0)
$$

where $a \equiv 2 \sqrt{2} G_{F} N_{e} E, G_{F}$ is Fermi constant and $N_{e}$ is the electron density in matter. In particular, taking the products of non-diagonal elements,

$$
\operatorname{Im}\left(\tilde{H}_{e \mu} \tilde{H}_{\mu \tau} \tilde{H}_{\tau e}\right)=\operatorname{Im}\left(H_{e \mu} H_{\mu \tau} H_{\tau e}\right)
$$

one obtains the following identity, which we call Naumov-Harrison-Scott identity,

$$
\tilde{\Delta}_{12} \tilde{\Delta}_{23} \tilde{\Delta}_{31} \tilde{J}=\Delta_{12} \Delta_{23} \Delta_{31} J
$$

in CP-odd part, where $\Delta_{i j} \equiv m_{i}^{2}-m_{j}^{2}, J \equiv \operatorname{Im} J_{e \mu}^{12}$ is Jarlskog factor [20], $J_{\alpha \beta}^{i j} \equiv U_{\alpha i} U_{\beta i}^{*} U_{\alpha j}^{*} U_{\beta j}$ and $U$ is the Maki-Nakagawa-Sakata (MNS) matrix [21]. Here the quantities expressed by the tilde include the matter effects. From this identity, $\tilde{J}$ can be expressed in terms of effective masses and the parameters of the Hamiltonian in vacuum. As effective masses shown in [15, 16, 17] do not depend on the CP phase, $\tilde{J}$ can be completely expressed by the linear term in $\sin \delta$. The reason why the CP dependence becomes simple is that $\tilde{J}$ is the products of four $\tilde{U}$ 's. Complicated matter effects included in a $\tilde{U}$ are partially canceled in $\tilde{J}$. 1

In this letter, we calculate $\tilde{U} \tilde{U}^{*}$. We use some matter invariant identities such as NaumovHarrison-Scott identity and express not only $\tilde{J}$ but also Re $\tilde{J}_{e \mu}^{i j}$ with the effective masses and the parameters in vacuum. The exact formula obtained in this method is very simple and the matter effects come in only through effective masses. We show that $\operatorname{Re} \tilde{J}_{e \mu}^{i j}$ has only a linear term in $\cos \delta$. That is, we prove that the oscillation probability in matter can be written in the form

$$
P\left(\nu_{e} \rightarrow \nu_{\mu}\right)=A \cos \delta+B \sin \delta+C
$$

without any approximation.

Another merit of our result is that the exact formula immediately reduces to the well-known approximate formulae both in low-energy [7] and in high-energy [10, 11]. We demonstrate that the approximate formula in high-energy can be easily reproduced from our exact formula as an example. Finally, we numerically calculate the coefficients $A, B$ and $C$.

\footnotetext{
${ }^{1}$ The calculation of a $\tilde{U}$ is performed by diagonalizing $\tilde{H}$ in Ref. [17].
} 


\section{Exact Formula of the Oscillation Probability}

The flavor and mass eigenstates are related by the MNS matrix $\tilde{U}_{\alpha i}$ in matter, where $\alpha=e, \mu, \tau$ is the flavor index, $i=1,2,3$ is the mass index. The amplitude for $\nu_{e}$ to $\nu_{\mu}$ transition is given by

$$
A\left(\nu_{e} \rightarrow \nu_{\mu}\right)=\sum_{i=1}^{3} \tilde{U}_{e i}^{*} e^{-i \frac{\lambda_{i}}{2 E} L} \tilde{U}_{\mu i}
$$

and the oscillation probability is also given by

$$
P\left(\nu_{e} \rightarrow \nu_{\mu}\right)=\left|A\left(\nu_{e} \rightarrow \nu_{\mu}\right)\right|^{2},
$$

from the amplitude, where $L$ stands for the baseline length.

We note from (5) that the amplitude depends only on the products $\tilde{U}_{e i} \tilde{U}_{\mu i}^{*}$. One of the important points in this letter is that these products can be easily calculated from identities which we derive below.

From the unitarity relation and the other two relations,

$$
\begin{aligned}
\tilde{H}_{e \mu} & =H_{e \mu}=p /(2 E), \\
\tilde{H}_{e \tau} \tilde{H}_{\tau \mu}-\tilde{H}_{e \mu} \tilde{H}_{\tau \tau} & =H_{e \tau} H_{\tau \mu}-H_{e \mu} H_{\tau \tau}=q /(2 E)^{2},
\end{aligned}
$$

three identities on the products $\tilde{U}_{e i} \tilde{U}_{\mu i}^{*}$ can be obtained as follows:

$$
\begin{aligned}
\sum_{i=1}^{3} \tilde{U}_{e i} \tilde{U}_{\mu i}^{*} & =\sum_{i=1}^{3} U_{e i} U_{\mu i}^{*}=0, \\
\sum_{i=1}^{3} \lambda_{i} \tilde{U}_{e i} \tilde{U}_{\mu i}^{*} & =\sum_{i=1}^{3} m_{i}^{2} U_{e i} U_{\mu i}^{*}=p, \\
\sum_{(i j k)}^{\text {cyclic }} \lambda_{j} \lambda_{k} \tilde{U}_{e i} \tilde{U}_{\mu i}^{*} & =\sum_{(i j k)}^{\text {cyclic }} m_{j}^{2} m_{k}^{2} U_{e i} U_{\mu i}^{*}=q,
\end{aligned}
$$

where $p$ and $q$ are constants determined by the parameters in vacuum and the sum is over $(i j k)=(123),(231),(312)$. We use the relation $\tilde{U}_{\tau i} \tilde{U}_{e j}-\tilde{U}_{e i} \tilde{U}_{\tau j}=\tilde{U}_{\mu k}^{*}(\operatorname{det} \tilde{U})$ etc, obtained from the formula $\tilde{U}^{\dagger}=\tilde{U}^{-1}=\tilde{\mathcal{U}}(\operatorname{det} \tilde{U})^{-1}$, where $\tilde{\mathcal{U}}$ represents the cofactor matrix.

Solving the simultaneous equations for the products $\tilde{U}_{e i} \tilde{U}_{\mu i}^{*}$, we obtain

$$
\tilde{U}_{e i} \tilde{U}_{\mu i}^{*}=\frac{p \lambda_{i}+q}{\tilde{\Delta}_{j i} \tilde{\Delta}_{k i}}
$$

where $(i j k)$ takes $(123),(231),(312)$. From the definition $\tilde{J}_{e \mu}^{i j}=\tilde{U}_{e i} \tilde{U}_{\mu i}^{*}\left(\tilde{U}_{e j} \tilde{U}_{\mu j}^{*}\right)^{*}$, the exact formula of the oscillation probability is given by

$$
P\left(\nu_{e} \rightarrow \nu_{\mu}\right)=-4 \sum_{(i j)}^{\text {cyclic }} \operatorname{Re} \tilde{J}_{e \mu}^{i j} \sin ^{2}\left(\frac{\tilde{\Delta}_{i j} L}{4 E}\right)-2 \sum_{(i j)}^{\text {cyclic }} \tilde{J} \sin \left(\frac{\tilde{\Delta}_{i j} L}{2 E}\right)
$$


where the sum is over $(i j)=(12),(23),(31)$ and

$$
\begin{aligned}
\operatorname{Re} \tilde{J}_{e \mu}^{i j} & =\frac{|p|^{2} \lambda_{i} \lambda_{j}+|q|^{2}+\operatorname{Re}\left(p q^{*}\right)\left(\lambda_{i}+\lambda_{j}\right)}{\tilde{\Delta}_{i j} \tilde{\Delta}_{12} \tilde{\Delta}_{23} \tilde{\Delta}_{31}} \\
\tilde{J} & =\frac{\operatorname{Im}\left(p q^{*}\right)}{\tilde{\Delta}_{12} \tilde{\Delta}_{23} \tilde{\Delta}_{31}} .
\end{aligned}
$$

We find that the matter effects are confined in the effective masses only. We can obtain the probability for antineutrinos, $\bar{\nu}_{e} \rightarrow \bar{\nu}_{\mu}$, by exchanging $a \rightarrow-a$ and $\delta \rightarrow-\delta$ in $\tilde{\Delta}_{i j}$ and $\tilde{J}_{e \mu}^{i j}$ of Eq. (13).

Let us comment on the relation between our result and that of other authors. The second identity (10) is also given in Ref. [22]. The third identity (11) is new and play an important role in deriving our exact formula. The similar expression to (12) is given in Ref. [18] as the result of the calculation of $e^{-i \tilde{H} L}$, although the CP phase has not been considered. 1 Next, $\operatorname{Im}\left(p q^{*}\right)$ in (15) are rewritten as

$$
\operatorname{Im}\left(p q^{*}\right)=1 /(2 E)^{3} \operatorname{Im}\left(H_{e \mu} H_{\mu \tau} H_{\tau e}\right)=\Delta_{12} \Delta_{23} \Delta_{31} J
$$

from (7) and (8). Naumov-Harrison-Scott identity is reproduced by substituting (16) into (15).

\section{Separation of CP odd/even Parts}

In this section, we give a concrete expression for the oscillation probability and then, we study the dependence of the oscillation probability on the CP phase.

First let us consider the constants $p$ and $q$. We use the standard parametrization

$$
U_{\alpha i}=\left(\begin{array}{ccc}
c_{13} c_{12} & c_{13} s_{12} & s_{13} e^{-i \delta} \\
-c_{23} s_{12}-s_{23} s_{13} c_{12} e^{i \delta} & c_{23} c_{12}-s_{23} s_{13} s_{12} e^{i \delta} & s_{23} c_{13} \\
s_{23} s_{12}-c_{23} s_{13} c_{12} e^{i \delta} & -s_{23} c_{12}-c_{23} s_{13} s_{12} e^{i \delta} & c_{23} c_{13}
\end{array}\right)
$$

where $\sin \theta_{i j}=s_{i j}, \cos \theta_{i j}=c_{i j}$. In addition, as the neutrino oscillation probabilities do not depend on the mass itself, but the mass square differences, we take $m_{1}^{2}=0, m_{2}^{2}=\Delta_{21}$ and $m_{3}^{2}=\Delta_{31}$ without loss of generality. So, $p$ and $q$ are given by

$$
p=p_{1} e^{-i \delta}+p_{2}, \quad q=q_{1} e^{-i \delta}+q_{2},
$$

where $p_{i}$ and $q_{i}$ are real numbers;

$$
\begin{aligned}
& p_{1}=\left(\Delta_{31}-\Delta_{21} s_{12}^{2}\right) s_{23} s_{13} c_{13}, \quad p_{2}=\Delta_{21} s_{12} c_{12} c_{23} c_{13}, \\
& q_{1}=-\Delta_{31} \Delta_{21} c_{12}^{2} s_{23} s_{13} c_{13}, \quad q_{2}=-\Delta_{31} \Delta_{21} s_{12} c_{12} c_{23} c_{13} .
\end{aligned}
$$

\footnotetext{
${ }^{2}$ We notice that the expression of $\tilde{U} \tilde{U}^{*}$ in $(12)$ is also derived from Eq. (4) in Ref. 23. after some calculations [24.
} 
Then, we have

$$
\begin{aligned}
|p|^{2} & =p_{1}^{2}+p_{2}^{2}+2 p_{1} p_{2} \cos \delta \\
|q|^{2} & =q_{1}^{2}+q_{2}^{2}+2 q_{1} q_{2} \cos \delta, \\
\operatorname{Re}\left(p q^{*}\right) & =p_{1} q_{1}+p_{2} q_{2}+\left(p_{1} q_{2}+q_{1} p_{2}\right) \cos \delta \\
\operatorname{Im}\left(p q^{*}\right) & =\left(p_{2} q_{1}-p_{1} q_{2}\right) \sin \delta .
\end{aligned}
$$

Therefore, the oscillation probability can be written in the form

$$
P\left(\nu_{e} \rightarrow \nu_{\mu}\right)=A \cos \delta+B \sin \delta+C,
$$

from (13)-(15). Note that $A, B$ and $C$ are independent of $\delta$ and the oscillation probability is expressed only by the linear terms in $\cos \delta$ and $\sin \delta$ up to a constant as described below. This is one of our main results. Here

$$
\begin{aligned}
& A=\sum_{(i j)}^{\text {cyclic }} A_{i j} \sin ^{2}\left(\frac{\tilde{\Delta}_{i j} L}{4 E}\right), \\
& B=\sum_{(i j)}^{\text {cyclic }} B^{\prime} \sin \left(\frac{\tilde{\Delta}_{i j} L}{2 E}\right), \\
& C=\sum_{(i j)}^{\text {cyclic }} C_{i j} \sin ^{2}\left(\frac{\tilde{\Delta}_{i j} L}{4 E}\right),
\end{aligned}
$$

are expressed by the products of the oscillation part dependent on $L$ and $A_{i j}, B^{\prime}$ and $C_{i j}$. And then, $A_{i j}, B^{\prime}$ and $C_{i j}$ are given by

$$
\begin{aligned}
A_{i j} & =\frac{-4\left[2 p_{1} p_{2} \lambda_{i} \lambda_{j}+2 q_{1} q_{2}+\left(p_{1} q_{2}+q_{1} p_{2}\right)\left(\lambda_{i}+\lambda_{j}\right)\right]}{\tilde{\Delta}_{i j} \tilde{\Delta}_{12} \tilde{\Delta}_{23} \tilde{\Delta}_{31}}, \\
B^{\prime} & =\frac{-2\left(p_{2} q_{1}-p_{1} q_{2}\right)}{\tilde{\Delta}_{12} \tilde{\Delta}_{23} \tilde{\Delta}_{31}} \\
C_{i j} & =\frac{-4\left[\left(p_{1}^{2}+p_{2}^{2}\right) \lambda_{i} \lambda_{j}+\left(q_{1}^{2}+q_{2}^{2}\right)+\left(p_{1} q_{1}+q_{2} p_{2}\right)\left(\lambda_{i}+\lambda_{j}\right)\right]}{\tilde{\Delta}_{i j} \tilde{\Delta}_{12} \tilde{\Delta}_{23} \tilde{\Delta}_{31}},
\end{aligned}
$$

as the function of the masses and mixing angles. Since the effective masses $\lambda_{i}$ shown in [15, 16, 17] do not depend on $\delta$, the coefficients $A, B$ and $C$ are independent of $\delta$.

Our analytic result given in (25) should be compared with the result of [12] depicted in Fig. 1 obtained numerically. The trajectory becomes an ellipse in the bi-probability space when $\delta$ changes from 0 to $2 \pi$. The CP dependence of the exact form of $P\left(\nu_{e} \rightarrow \nu_{\mu}\right)$ given in (25) becomes much simpler than the result in [16]. By solving (25) for $\sin \delta$ and $\cos \delta$ one obtain

$$
\sin \delta=\frac{B(P-C) \pm A \sqrt{A^{2}+B^{2}-(P-C)^{2}}}{A^{2}+B^{2}}
$$




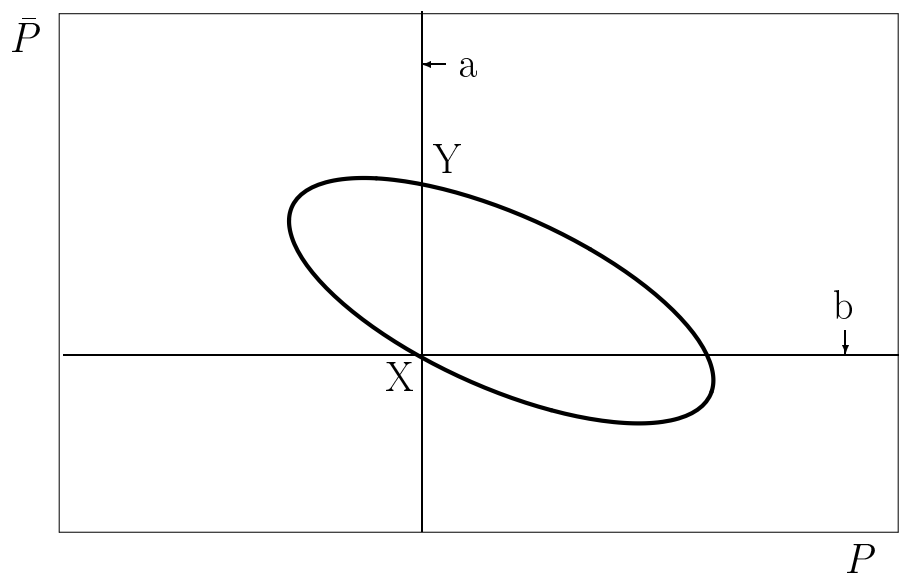

Figure 1: An example of CP trajectory We take $P$ for the horizontal axis and $\bar{P}$ for the vertical axis. The value of $\delta$ changes from 0 to $2 \pi$.

$$
\cos \delta=\frac{A(P-C) \mp B \sqrt{A^{2}+B^{2}-(P-C)^{2}}}{A^{2}+B^{2}} .
$$

Thus, we can determine the value of CP phase except for the ambiguity of the sign from the measurement of the probability. The sign ambiguity is understood as follows. If we measure the probability of the neutrino at a fixed energy and a baseline, we find the solutions on a "line a". As shown in Fig. 1, there are two intersections X and Y of "line a" with the CP trajectory. This is the reason why the ambiguity due to the sign appears in the analytic solutions (32) and (33).

In order to resolve the sign ambiguity, we need to measure more than two kinds of probabilities, for example, neutrino and antineutrino. We denote $P$ and $\bar{P}$ of the oscillation probabilities for neutrino and antineutrino respectively as

$$
\begin{aligned}
P & =A \cos \delta+B \sin \delta+C, \\
\bar{P} & =\bar{A} \cos \delta+\bar{B} \sin \delta+\bar{C} .
\end{aligned}
$$

Then, CP phase can be determined by

$$
\begin{aligned}
\sin \delta & =\frac{(\bar{A} P-A \bar{P})-(\bar{A} C-A \bar{C})}{\bar{A} B-A \bar{B}} \\
\cos \delta & =\frac{(\bar{B} P-B \bar{P})-(\bar{B} C-B \bar{C})}{\bar{B} A-B \bar{A}}
\end{aligned}
$$

without the ambiguity of the sign. This means that the solution is at X, the intersect of "line a" and "line b".

Although the value of $\mathrm{CP}$ phase is determined, in principle, in (36) and (37), there remain other ambiguities included in $A, B, C$ and $\bar{A}, \bar{B}, \bar{C}$. The methods to resolve these ambiguities are discussed in the references for example [12, 25, 26, 27]. We discuss the ambiguities due to the sign of mass squared differences in Sec. 5 . 


\section{Simple Derivation of Approximate Formula}

In the previous section, we have shown that the exact formula of the oscillation probability can be expressed as $P\left(\nu_{e} \rightarrow \nu_{\mu}\right)=A \cos \delta+B \sin \delta+C$. In this section, we demonstrate that the approximate formula seen in 10, 11] is easily derived as an example in the case of $m_{1}<m_{2} \ll m_{3}$. One obtains the approximate formula for other patterns of mass hierarchy in the same way.

Let us first consider the coefficient $B$ of $\sin \delta$. $B$ is expressed in the form of the sum as (27). Note that, under the condition $x+y+z=0$, the identity

$$
\sin 2 x+\sin 2 y+\sin 2 z=-4 \sin x \sin y \sin z,
$$

holds, and $B$ from (27) is rewritten in the form of product as

$$
\begin{aligned}
B & =\sum_{(i j)}^{\text {cyclic }} B^{\prime} \sin \left(\frac{\tilde{\Delta}_{i j} L}{2 E}\right) \\
& =-4 B^{\prime} \sin \left(\frac{\tilde{\Delta}_{12} L}{4 E}\right) \sin \left(\frac{\tilde{\Delta}_{23} L}{4 E}\right) \sin \left(\frac{\tilde{\Delta}_{31} L}{4 E}\right) .
\end{aligned}
$$

Next, let us consider the coefficient $A$ of $\cos \delta$. Under the same condition as in deriving $B$, the identity

$$
\sin ^{2} x=-(\sin x \sin y \cos z+\sin x \cos y \sin z)
$$

holds and $A$ is rewritten as

$$
\begin{aligned}
A & =\sum_{(i j)}^{\text {cyclic }} A_{i j} \sin ^{2}\left(\frac{\tilde{\Delta}_{i j} L}{4 E}\right) \\
& =-\sum_{(i j k)}^{\text {cyclic }}\left(A_{j k}+A_{k i}\right) \cos \left(\frac{\tilde{\Delta}_{i j} L}{4 E}\right) \sin \left(\frac{\tilde{\Delta}_{j k} L}{4 E}\right) \sin \left(\frac{\tilde{\Delta}_{k i} L}{4 E}\right) .
\end{aligned}
$$

Substituting (19) and (20) for $p$ and $q$ in (29)-(31), $A, B$ and $C$ are rewritten with the masses and the mixings as

$$
\begin{aligned}
& A=\sum_{(i j k)}^{\text {cyclic }} \frac{-8 J_{r} \Delta_{21}\left[\Delta_{31} \lambda_{k}\left(\lambda_{k}-\Delta_{31}\right)+A_{k}^{(1)}\right]}{\tilde{\Delta}_{j k}^{2} \tilde{\Delta}_{k i}^{2}} \cos \left(\frac{\tilde{\Delta}_{i j} L}{4 E}\right) \sin \left(\frac{\tilde{\Delta}_{j k} L}{4 E}\right) \sin \left(\frac{\tilde{\Delta}_{k i} L}{4 E}\right), \\
& B=\frac{8 \Delta_{12} \Delta_{23} \Delta_{31}}{\tilde{\Delta}_{12} \tilde{\Delta}_{23} \tilde{\Delta}_{31}} J_{r} \sin \left(\frac{\tilde{\Delta}_{12} L}{4 E}\right) \sin \left(\frac{\tilde{\Delta}_{23} L}{4 E}\right) \sin \left(\frac{\tilde{\Delta}_{31} L}{4 E}\right), \\
& C=\sum_{(i j)}^{\operatorname{cyclic}} \frac{-4\left[s_{13}^{2}\left(s_{23}^{2} c_{13}^{2} \Delta_{31}^{2} \lambda_{i} \lambda_{j}+C_{i j}^{(1)}+C_{i j}^{(2 a)}\right)+C_{i j}^{(2 b)}\right]}{\tilde{\Delta}_{i j} \tilde{\Delta}_{12} \tilde{\Delta}_{23} \tilde{\Delta}_{31}} \sin ^{2}\left(\frac{\tilde{\Delta}_{i j} L}{4 E}\right),
\end{aligned}
$$


where $J_{r}=s_{12} c_{12} s_{23} c_{23} s_{13} c_{13}^{2}$, and

$$
\begin{aligned}
A_{k}^{(1)} & =\Delta_{21}\left\{\Delta_{31} \lambda_{k}\left(c_{12}^{2}-s_{12}^{2}\right)+\lambda_{k}^{2} s_{12}^{2}-\Delta_{31}^{2} c_{12}^{2}\right\} \\
C_{i j}^{(1)} & =\Delta_{21} \Delta_{31}\left\{-\lambda_{i}\left(\lambda_{j} s_{12}^{2}+\Delta_{31} c_{12}^{2}\right)-\lambda_{j}\left(\lambda_{i} s_{12}^{2}+\Delta_{31} c_{12}^{2}\right)\right\} s_{23}^{2} c_{13}^{2}, \\
C_{i j}^{(2 a)} & =\Delta_{21}^{2}\left(\lambda_{i} s_{12}^{2}+\Delta_{31} c_{12}^{2}\right)\left(\lambda_{j} s_{12}^{2}+\Delta_{31} c_{12}^{2}\right) s_{23}^{2} c_{13}^{2}, \\
C_{i j}^{(2 b)} & =\Delta_{21}^{2}\left(\lambda_{i}-\Delta_{31}\right)\left(\lambda_{j}-\Delta_{31}\right) s_{12}^{2} c_{12}^{2} c_{23}^{2} c_{13}^{2} .
\end{aligned}
$$

Note that these expressions are still exact. In the limit of small $\Delta_{21}$, terms given in Eqs. (47)-(50) are higher order in $\Delta_{21}$ and can be ignored. The superscripts of $A$ and $C$ stand for the power of $\Delta_{21}$, and (2a) represents the term proportional to $s_{13}^{2}$ and $(2 b)$ is the term independent of $s_{13}^{2}$.

Finally, we obtain the well known approximate formula by neglecting the smallest effective mass. In the high energy neutrino the smallest effective mass is $\lambda_{1} \simeq \Delta_{21}$. Other effective masses $\lambda_{2}$ and $\lambda_{3}$, correspond to $a$ or $\Delta_{31}$. Accordingly, $A, B$ and $C$ are approximated by

$$
\begin{aligned}
& A=\frac{8 J_{r} \Delta_{21} \Delta_{31}}{a\left(\Delta_{31}-a\right)} \cos \left(\frac{\Delta_{31} L}{4 E}\right) \sin \left(\frac{a L}{4 E}\right) \sin \left(\frac{\left(\Delta_{31}-a\right) L}{4 E}\right) \\
& B=\frac{8 J_{r} \Delta_{21} \Delta_{31}}{a\left(\Delta_{31}-a\right)} \sin \left(\frac{\Delta_{31} L}{4 E}\right) \sin \left(\frac{a L}{4 E}\right) \sin \left(\frac{\left(\Delta_{31}-a\right) L}{4 E}\right) \\
& C=\frac{4 \Delta_{31}^{2}}{\left(\Delta_{31}-a\right)^{2}} s_{23}^{2} s_{13}^{2} c_{13}^{2} \sin ^{2}\left(\frac{\left(\Delta_{31}-a\right) L}{4 E}\right)
\end{aligned}
$$

under the condition $\Delta_{21} / \Delta_{31}<s_{13}$. When $s_{13}$ is smaller than $\left(\Delta_{21} / \Delta_{31}\right)$, the term $C_{i j}^{(2 b)}$ independent of $s_{13}$ becomes the dominant term. Although the approximate formula derived here is in agreement with the ones seen in [10, 11], the derivation is rather simple. Moreover, one can easily reproduce the approximate formula in low-energy [7].

\section{Numerical Analysis of CP odd/even Part}

In this section, we investigate the values of the coefficients $A, B$ and $C$ in cases of neutrino and antineutrino using the exact expressions. We also investigate them changing the signs of $\Delta_{31}$ and $\Delta_{21}$.

In this numerical analysis, we take $\theta_{12}=\pi / 4,\left|\Delta_{21}\right|=10^{-4} \mathrm{eV}^{2}, \theta_{23}=\pi / 4$ and $\left|\Delta_{31}\right|=$ $3 \times 10^{-3} \mathrm{eV}^{2}$ to be consistent with the LMA MSW solution to the solar neutrino problem [2, 3] and the zenith-angle dependences of atmospheric neutrinos [1]. We also take $\theta_{13}=0.05$ within the upper bound of CHOOZ experiment [29]. The baseline length is typically taken to be $L=2900 \mathrm{~km}$ and the matter density is taken to be $3.2 \mathrm{~g} / \mathrm{cm}^{3}$.

In Fig. 2 we show the coefficients $A, B$ and $C$ changing with the energy $E$. We observe that the sign of $A$ is opposite for example in Fig. 2(a) and (d). We also observe that $A$ and $B$ have the opposite sign but $C$ has the same sign comparing Fig. 2(a) with (e). In addition, some peaks have appeared in all graphs of Fig. 2 with the change of energy. In case of $\Delta_{31}>0$, the peaks 

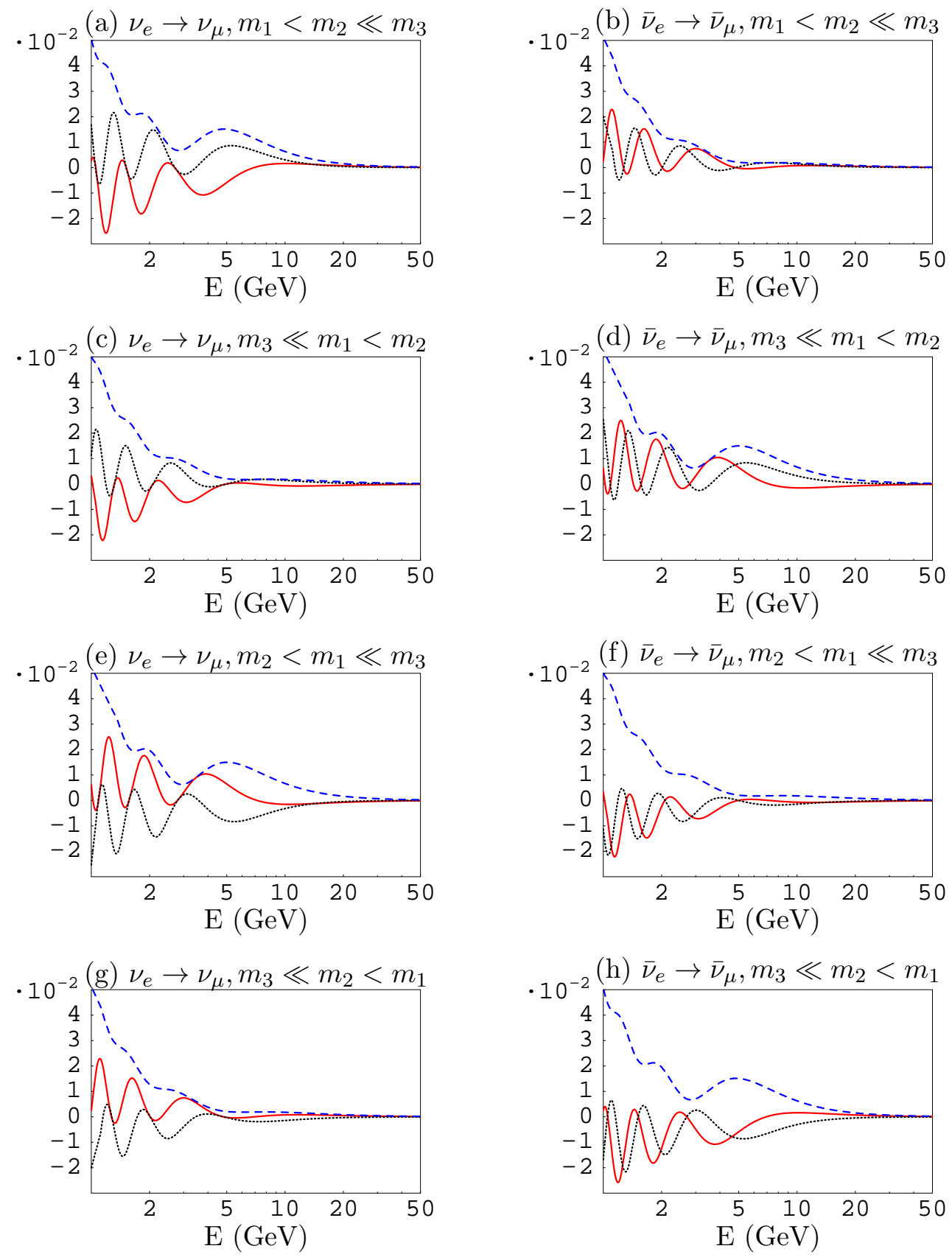

Figure 2: $A, B, C$ at $L=2900 \mathrm{~km}$ The graphs of the left and right side correspond to the neutrino and the antineutrino respectively. The solid lines, the dotted lines and the dashed lines are for $A, B$ and $C$ in all graphs. And from top to bottom, $\left(\Delta_{31}>0, \Delta_{21}>0\right),\left(\Delta_{31}<0, \Delta_{21}>0\right)$, $\left(\Delta_{31}>0, \Delta_{21}<0\right)$ and $\left(\Delta_{31}<0, \Delta_{21}<0\right)$ cases. 
around $6 \mathrm{GeV}$ in Fig. 2(a) for neutrinos are enhanced compared with those in Fig. 2(b) for antineutrinos. Inversely, in case of $\Delta_{31}<0$, the peaks in Fig. 2(d) for antineutrinos are enhanced compared with those in Fig. 2(c) for neutrinos.

These features are understood qualitatively from the approximate formula (51)-(53). First let us consider the sign of $A, B$ and $C$. As we found from (51)-(53), when the signs of both $\Delta_{31}$ and a change, the sign of $A$ becomes opposite and the signs of $B$ and $C$ do not change. On the other hand, when the sign of $\Delta_{21}$ changes, the signs of both $A$ and $B$ change while the sign of $C$ does not change. Next, let us consider the magnitude of the peak around $6 \mathrm{GeV}$. These are strongly affected by the denominator $\left(\Delta_{31}-a\right)$. Since the signs of $\Delta_{31}$ and $a$ are opposite in Fig. 2(a) and $(\mathrm{d})$, the denominator $\left(\Delta_{31}-a\right)$ becomes small and the magnitude of the peaks are enhanced. On the other hand, since the signs of $\Delta_{31}$ and $a$ are the same in Fig. 2(b) and (c), the peaks are suppressed. Finally, let us explain the position of the peak in Fig. 2(a) and (d) around $6 \mathrm{GeV}$. Roughly, the peak energy is determined by the following:

$$
\sin \left[1.27\left(\frac{\Delta_{31}-a}{1 \mathrm{eV}^{2}}\right)\left(\frac{L}{1 \mathrm{~km}}\right)\left(\frac{E}{1 \mathrm{GeV}}\right)^{-1}\right] \sim 1 \rightarrow E \simeq 6 \mathrm{GeV} \quad(\text { at } L=2900 \mathrm{~km})
$$

As pointed out by Parke and Weiler [8], and Lipari [13], the peak energy is lower than the energy of 1-3 MSW resonance since the baseline length is short compared with the earth diameter. The above discussions on Fig. 2(a)-(e) can be applied to other figures.

We have studied how the magnitude of $A, B$ and $C$ change due to the sign of the mass squared differences. In the case of $m_{1}<m_{2} \ll m_{3}$, the coefficients have been investigated in Ref. [10] by using the approximate formula. These correspond to Fig. 2(a) and (b). The sign of $\Delta_{31}$ is determined from the leading term $C$ as pointed out by many authors (for example see [10]). On the other hand, the sign of $\Delta_{21}$ is determined from next leading terms $A$ or $B$. This means that the sign of $\Delta_{21}$ is simultaneously determined in addition to the CP phase. It may be interesting as the first observation of the sign of $\Delta_{21}$ using artificial neutrino beam.

\section{Summary}

We have studied neutrino oscillations in constant matter within the framework of the three neutrino scenario. We summarize the results obtained in this letter.

(i) We have derived an exact expression of the oscillation probability by using a new method. We have calculated $\tilde{U} \tilde{U}^{*}$ from the identities without directly calculating single $\tilde{U}$. Not only the derivation but also the result becomes simple and the matter effects enter only through effective masses.

(ii) We have obtained the CP dependence of the oscillation probability exactly by using the standard parametrization. It has been shown that the oscillation probability is in the form, $P\left(\nu_{e} \rightarrow \nu_{\mu}\right)=A \cos \delta+B \sin \delta+C$. We have also demonstrated that the approximate formula in high-energy can be easily reproduced from our result. 
Finally, let us comment on the oscillation probabilities for other channels. These probabilities are easily derived in the same way as $P\left(\nu_{e} \rightarrow \nu_{\mu}\right) . P\left(\nu_{e} \rightarrow \nu_{\tau}\right)$ has the same CP dependence as $P\left(\nu_{e} \rightarrow \nu_{\mu}\right)$. However, $P\left(\nu_{\mu} \rightarrow \nu_{\tau}\right)$ has the term which depends on $\cos 2 \delta$ in addition to the linear terms in $\sin \delta$ and $\cos \delta$.

\section{Acknowledgement}

The authors would like to thank Prof. A. I. Sanda for reading the manuscript and making a number of helpful suggestions. We would like to thank Prof. H. Minakata and Prof. O. Yasuda for discussions and valuable comments.

\section{References}

[1] Super-Kamiokande Collaboration, Y. Fukuda et al., Phys. Rev. Lett. 81, (1998) 1562; Phys. Lett. B433, (1998) 9; Phys. Lett. B436, (1998) 33; Phys. Rev. Lett. 82, (1999) 2644.

[2] GALLEX Collaboration, W. Hampel et al., Phys. Lett. B447, (1999) 127; SAGE Collaboration, J. N. Abdurashitov et al., Phys. Rev. C60 (1999) 055801; Homestake Collaboration, B. T. Cleveland et al., Astrophys. J. 496, (1998) 505; Super-Kamiokande Collaboration, Y. Fukuda et al., Phys. Rev. Lett. 82, (1999) 1810, ibid. 82, (1999) 2430.

[3] SNO Collaboration, Q. R. Ahmad et al., Phys. Rev. Lett. 87, (2001) 071301.

[4] Y. Itow et al., hep-ex/0106019; N. Okamura, M. Aoki, K. Hagiwara, Y. Hayato, T. Kobayashi, T. Nakaya and K. Nishikawa, hep-ph/0104220; hep-ph/0112338.

[5] S. H. Geer, Phys. Rev. D57, (1998) 6989.

[6] P. F. Harrison and W. G. Scott, Phys. Lett. B476, (2000) 349.

[7] J. Arafune, M. Koike and J. Sato, Phys. Rev. D56, (1997) 3093; Erratum ibid., D60, (1999) 119905.

[8] S. J. Parke and T. J. Weiler, Phys. Lett. B501, (2001) 106.

[9] For example

J. Arafune and J. Sato, Phys. Rev. D55, (1997) 1653;

H. Minakata and H. Nunokawa, Phys. Lett. B413, (1997) 369, Phys. Rev. D57, (1998) 4403;

M. Bilenky, C. Giunti and W. Grimus, Phys. Rev. D58, (1998) 033001;

A. De Rujula, M. B. Gavela and P. Hernandez, Nucl. Phys. B547, (1999) 21;

K. Dick, M. Freund, M. Lindner and A. Romanino, Nucl. Phys. B562, (1999) 29;

M. Tanimoto, Phys. Lett. B462, (1999) 115;

A. Donini, M. B. Gavela, P. Hernandez and S. Rigolin, Nucl. Phys. B574, (2000) 23;

A. Romanino, Nucl. Phys. B574, (2000) 675; 
O. Yasuda, Acta Phys. Polon. B30, (1999) 3089;

J. Sato, Nucl. Instrum. Meth. A451, (2000) 36;

M. Koike and J. Sato, Phys. Rev. D61, (2000) 073012; Erratum ibid., D62, (2000) 079903;

H. Minakata and H. Nunokawa, Phys. Lett. B495, (2000) 369;

H. Yokomakura, K. Kimura and A. Takamura, Phys. Lett. B496, (2000) 175;

M. Koike, T. Ota and J. Sato, Phys. Rev. D65, (2002) 053015;

T. Miura, E. Takasugi, Y. Kuno and M. Yoshimura, Phys. Rev. D64, (2001) 013002;

I. Mocioiu and R. Shrock, JHEP 0111 (2001) 050;

J. Pinney and O. Yasuda, Phys. Rev. D64, (2001) 093008.

[10] A. Cervera, A. Donini, M. B. Gavela, J. J. Gomez Cadenas, P. Hernandez, O. Mena and S. Rigolin, Nucl. Phys. B579, (2000) 17 [Erratum-ibid. B593, (2000) 731].

[11] M. Freund, Phys. Rev. D64, (2001) 053003.

[12] H. Minakata and H. Nunokawa, JHEP 0110 (2001) 001.

[13] P. Lipari, Phys. Rev. D64, (2001) 033002.

[14] M. Freund, P. Huber and M. Lindner, Nucl. Phys. B615, (2001) 331.

[15] V. Barger, K. Whisnant, S. Pakvasa and R. J. N. Phillips, Phys. Rev. D22, (1980) 2718;

[16] H. W. Zaglauer and K. H. Schwarzer, Z. Phys. C40, (1988) 273.

[17] Z. Xing, Phys. Lett. B487, (2000) 327.

[18] T. Ohlsson and H. Snellman, J. Math. Phys. 41, (2000) 2768; Phys. Lett. B474, (2000) 153.

[19] V. A. Naumov, Int. J. Mod. Phys. D1, (1992) 379.

[20] C. Jarlskog, Phys. Rev. Lett. 55, (1985) 1039.

[21] Z. Maki, M. Nakagawa, S. Sakata, Prog. Theor. Phys. 28, (1962) 870.

[22] Z. Xing, Phys. Rev. D64, (2001) 033005.

[23] P. F. Harrison and W. G. Scott, hep-ph/0203021.

[24] P. F. Harrison, private communication.

[25] J. Burguet-Castell, M. B. Gavela, J. J. Gomez-Cadenas, P. Hernandez and O. Mena, Nucl. Phys. B608, (2001) 301.

[26] V. Barger, D. Marfatia and K. Whisnant, hep-ph/0112119.

[27] T. Kajita, H. Minakata and H. Nunokawa, Phys. Lett. B528, (2002) 245. 
[28] L. Wolfenstein, Phys. Rev. D17, (1978) 2369; D20, (1979) 2634; S. P. Mikheyev and A. Yu Smirnov, Sov. J. Nucl. Phys. 42, (1985) 913.

[29] CHOOZ Collaboration, M. Apollonio et al., Phys. Lett. B420, (1998) 397; Phys. Lett. B466, (1999) 415. 